\section{Cahiers de Narratologie}

Analyse et théorie narratives

8 | 1997

Création de l'espace et narration littéraire

\title{
Génétique du lieu romanesque
}

Sur quelques dessins de Zola

\section{Philippe Hamon}

\section{(2) OpenEdition}

Journals

Édition électronique

URL : http://journals.openedition.org/narratologie/10718

DOI : 10.4000/narratologie.10718

ISSN : $1765-307 X$

Éditeur

LIRCES

\section{Édition imprimée}

Date de publication : 1 décembre 1997

Pagination : 15-26

ISBN : 291089746X

ISSN : 0993-8516

Référence électronique

Philippe Hamon, « Génétique du lieu romanesque », Cahiers de Narratologie [En ligne], 8| 1997, mis en ligne le 01 décembre 2020, consulté le 25 février 2021. URL : http://journals.openedition.org/ narratologie/10718 ; DOI : https://doi.org/10.4000/narratologie.10718

Ce document a été généré automatiquement le 25 février 2021.

Article L.111-1 du Code de la propriété intellectuelle. 


\title{
Génétique du lieu romanesque
}

\author{
Sur quelques dessins de Zola
}

\section{Philippe Hamon}

1 La critique génétique a pour objet privilégié, on le sait depuis son institutionnalisation (I.T.E.M.), et avant celle-ci (travaux d'Albalat sur le «travail» de Flaubert), les brouillons et manuscrits préparatoires des écrivains (mais aussi des artistes au sens large : musiciens, architectes etc.). Les dossiers préparatoires de Zola, qui ont intéressé très tôt la critique, du vivant même de l'écrivain, et qui sont bien conservés (B. $\mathrm{N}$. Paris), ont déjà fait l'objet de nombreuses études (H. Massis, H. Mitterand, C. Becker...).

2 Les dossiers préparatoires des vingt Rougon-Macquart se ressemblent. Zola a tout fait pour cela, les classant et les conservant soigneusement pour en faire en quelque sorte la vitrine de la fameuse "méthode» naturaliste. Ils comportent en général des «sections» identiques: une «Ebauche» («soliloque que l'auteur tient avec lui même »), un fichier « Personnages », deux « Plans » rédigés chapitre par chapitre, des « Notes » (prises sur le terrain, ou prises d'après des livres techniques), des documents divers (coupures de presse, lettres de correspondants, photographies, cartes de visites d'informateurs), des listes (de noms propres, de noms de lieux, de titres "essayés », de dates), des plans et des dessins (de la main de Zola, ou de la main de certains de ses informateurs privilégiés, comme par exemple ceux de l'architecte F. Jourdain qui prépare pour Zola certains de ses "décors »). C'est de certains dessins de la main de Zola, dessins topographiques, que je voudrais dire quelques mots de commentaire ${ }^{2}$.

3 Une remarque générale auparavant : toute œuvre littéraire peut-être, et celle de Zola homme du XIXe siècle, sans doute, en particulier, est d'essence polysémiotique. Que ce soit en avant-texte, à son stade de germination, que ce soit au niveau du texte imprimé, ou que ce soit au niveau de sa lecture par un lecteur concret ou de sa diffusion et de ses récritures ultérieures, toute œuvre est, pour qui la conçoit ou la reçoit, une combinaison de signes (écrits), de consignes (de lecture, plus ou moins implicites ou explicites), d'indices et de symptômes (renvoyant à un extratexte biographique) et d'icônes (entités analogiques du réel qu'elles signifient: images, cartes, dessins, photographies, schémas, calligrammes, diagrammes, maquettes, etc. $\left.{ }^{3}\right)$. Ces dernières peuvent prendre des formes diverses, et intervenir dans la totalité d'un processus 
littéraire (ou non littéraire: voir les tableaux de Manet à l'origine des articles de critique d'art de Zola) à des moments divers de sa mise en œuvre : images publicitaires ou de tableaux, photographies (Zola est lui-même photographe, surtout après 1888) faisant partie de l'environnement immédiat de l'écrivain ${ }^{4}$, gravures de journaux, diagrammes, sollicitant et médiatisant l'inspiration ou assistant l'écriture en cours de tel roman (les diagrammes des divers arbres généalogiques de la famille des RougonMacquart ; une gravure de Breughel à l'origine du Ventre de Paris : une vue panoramique de Paris pour la préparation d'Une Page d'amour; les plans de Jourdain pour Le Rêve et Au bonheur des Dames), tableaux réels regardés par les personnages de l'œuvre (la noce de Gervaise visitant le Louvre dans L'Assommoir) ou tableaux fictifs peints par les personnages de peintres de tel ou tel roman (tableaux de Claude dans Le Ventre de Paris et dans L'Euvre, dessins de Clotilde dans Le Docteur Pascal), illustrations, couvertures illustrées, frontispices des éditions illustrées (voir les démêlés de Zola avec son illustrateur du Rêve, Carlos Schwabe, ou avec l'édition allemande illustrée de La Débâcle), mises au cinéma postérieures (Renoir, F. Lang, Clément), au théâtre (souvent par Zola lui-même), ou en affiches de librairie, de tel ou tel roman, tableaux (Manet ou Cézanne peignant Zola), reportages illustrés concernant l'écrivain (L'Illustration du 8 mars 1890 ; La Revue Illustrée du 15 février 1887, etc.), parodies illustrées et caricatures (Zola est sans doute, avec Hugo, l'écrivain le plus caricaturé du XIXe siècle ${ }^{5}$ ). L'iconique, au sens large, traverse le texte zolien et l'accompagne à tous les stades de son existence. Et une éventuelle critique génétique qui aborderait en particulier l'avanttexte zolien (mais sans doute, aussi, beaucoup d'autres ${ }^{6}$ ) serait d'emblée nulle et non advenue si elle ne prenait pas en considération l'ensemble du système poly-sémiotique (textuel, inter-textuel ${ }^{7}$, iconique, icôno-textuel, et inter-icônique) qui le conditionne, et notamment ces dessins, tracés, dans le texte même du dossier préparatoire, de la même main que la main qui trace à côté des mots et des phrases, dessins à référents à la fois intra-textuels et virtuels puisque, comme le plan de l'architecte, ils renvoient à une œuvre à venir, à une description ou à un récit qui n'existe pas encore, à un texte non encore écrit, ou en cours d'écriture, de l'écrivain.

Contrairement au roman d'aventures (type J. Verne) qui s'accompagne volontiers de plans et cartes, destinés au lecteur, contrairement au roman policier, qui souvent publie, dans le texte achevé, les plans des lieux (la chambre ou la maison du crime) pour soutenir la lecture et les hypothèses du lecteur (notamment dans le cas du genre $\mathrm{du}$ «meurtre dans un local clos de l'intérieur ", type Mystère de la chambre jaune), le texte zolien ne publiera pas ces plans des dossiers préparatoires, qui restent cantonnés à l'usage interne et privé de son avant-texte. Leur fonction essentielle est d'assister la création en cours, d'assurer, le temps que dure la mise en intrigue et la rédaction proprement dite du roman, la cohérence générale de ce dernier. Ce sont, pour l'écrivain écrivant, des sortes de « lieux de mémoire ", pour que, d'un chapitre à l'autre, telle rue continue de donner dans telle autre (plan de quartier), tel commerce reste contigu de tel autre (plan de rue), telle commode reste en face de telle porte (plan d'appartement), ou pour que, à l'intérieur d'un même chapitre, au cours par exemple de la description d'un dîner, tel personnage reste voisin de tel autre au cours du dîner (plan de table). Leur fonction n'est donc pas "réaliste", du moins au premier chef, même pour les plans qui renvoient à des lieux réels (la Bourse, tel quartier de Paris, etc.), mais d'assurer une sorte de «mémoire prospective » dans et de l'écriture en cours, à la fois structure d'appel et de rappel, rappelant la distribution des personnages, et suggérant leurs déplacements possibles, construisant des antagonismes ou des affinités de 
milieux, assurant une constante et une cohérence dans les descriptions non encore rédigées.

5 A peu près tous les dossiers préparatoires des Rougon-Macquart comportent, dessinés de la main de Zola, de tels dessins qui sont des plans de lieux ${ }^{8}$ : hôtel et serre de $L a$ Curée, plan du quartier de Passy et du jardin des Deberle (Une Page d'Amour), de la place de la Bourse (L'Argent), plan d'étages et d'appartements (Pot-Bouille), tracé d'une portion de ligne de chemin de fer entre Motteville et Rouen (La bête humaine), plans des étages des magasins du Bon Marché et du Louvre (Au bonheur des dames), du grand jardin du Paradou et de la campagne voisine (La faute de l'abbé Mouret), du cimetière de SaintOuen (L'Cuvre), du carreau et du quartier des Halles (Le ventre de Paris), plan des lieux de Germinal et de la propriété ou se passe Le Docteur Pascal, du quartier et de la demeure de Gervaise (L'assommoir), des villes de Chateaudun et de Cloyes, d'une grande ferme ( $L a$ Terre), de la ville de Plassans (La Fortune des Rougon), du champ de bataille et de la chambre de Napoléon III (La Débâcle), plan de la table du souper de Nana avec places des personnages (Nana) etc.

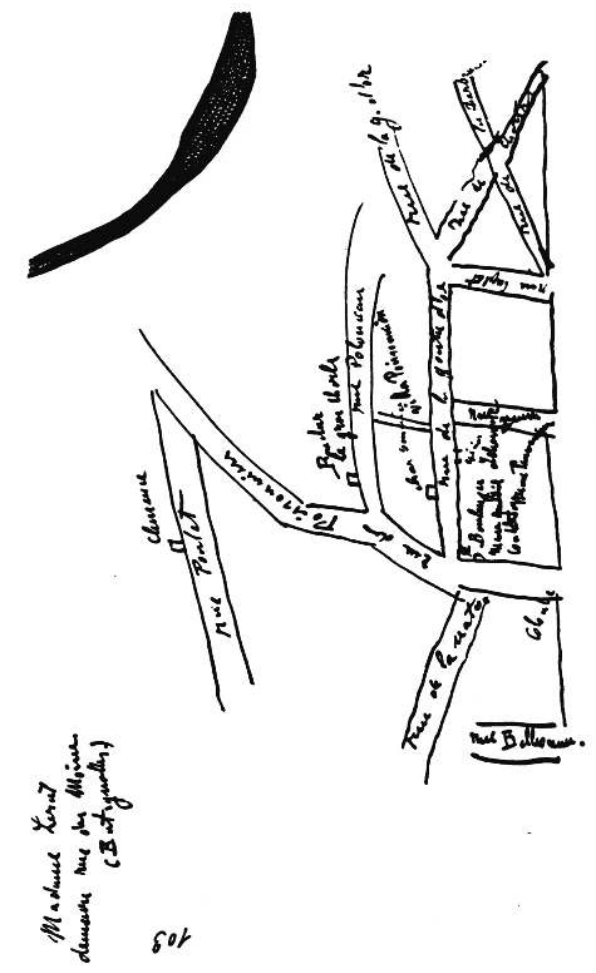

L'Assommoir : La rue de la Goutte-d'Or et les alentours. Plan dessiné par l'auteur (B.N. Ms. n.a.f. 10.271, $f^{\circ}$ 103). 


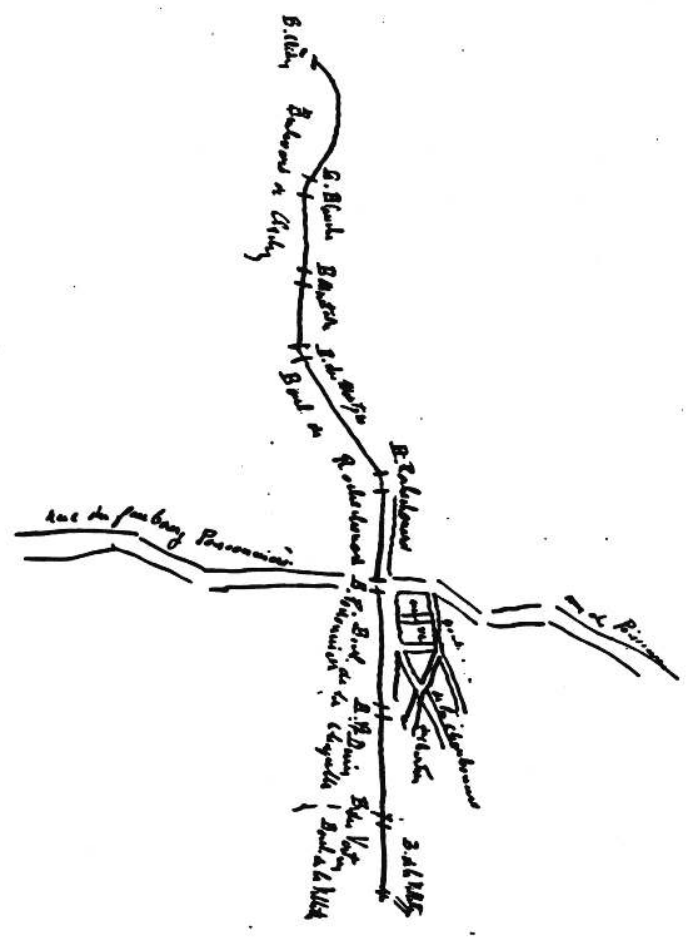

L'Assommoir : Plan général du quartier, dessiné par l'auteur (B.N. Ms. N.A.F. 10.271, f 104).

6 Sauf exception, ces dessins, à la plume ou au crayon (le crayon signalant en général un dessin fait « sur le terrain »), qui sont souvent surchargés ou accompagnés en marge de notes (noms de rues, dimensions ou distances, noms de personnages ou de lieux, paraphrases du croquis), sont tous des plans. Pas de portraits de personnages, pas d'élévation ni de coupe de bâtiment ${ }^{9}$, pas de dessin d'objet ou de meuble ${ }^{10}$, pas de détails d'habillement, pas de paysage ou de vue perspective. Comme si l'imaginaire iconique de l'espace, in statu nascendi, était, chez Zola, dans ces croquis qui soutiennent et accompagnent la rédaction du roman, un monde sans volumétrie à deux dimensions, planaire et cadastral, « à vol d'oiseau », le plus « abstrait » qui soit (si on le compare par exemple à la perspective, ou à la vue axonométrique, plus « réalistes»), composé de « lignes » (et parfois même réductible à une seule ligne, comme cette portion de chemin de fer du dossier préparatoire de La Bête humaine). Peu importe que certains de ces dessins tracent le plan de lieux réduits (une table, une chambre, une serre, le jardin d'une maison), d'autres de lieux vastes (une ville), que certains servent à assister la rédaction d'une brève description unique ou d'autres servent à assister l'économie spatiale globale du roman tout entier, que certains tracent le plan de lieux fictifs (les parcelles de La Terre, le jardin et les environs du Paradou, le petit port de La joie de vivre, la Souléiade du Docteur Pascal, la ville du Rêve), d'autres le plan de lieux réels (le quartier des Halles, de la Goutte d'Or, la place de la Bourse, Passy, une grande ferme dans la Beauce, le magasin du Bon Marché), d'autres le plan de lieux semi-réels (ou semi-fictifs : le plan d'Aix-Plassans, celui de Rognes-Romilly pour La Terre, sans doute de nombreux plans d'appartements ou de jardins, comme dans les dossiers préparatoires de Pot-Bouille ou d'Une page d'amour) : leur fonction de distribution du personnel romanesque, de soutien mémoriel et prospectif à l'écriture en cours est identique. 
7 Plusieurs tendances peuvent se laisser observer, que je me contenterai d'indiquer rapidement: la première consiste, pour Zola, à dessiner pour chaque roman en préparation une série de plans qui, par focalisation progressive, passent du plus global au plus local, d'une grande échelle à une échelle plus réduite, d'un plan général (plan de ville, de quartier de ville) à des plans de lieux de plus en plus précis : plan du site général ou du quartier, plan d'un groupe de rues, plan d'un immeuble, ou d'une maison, plan d'un étage, plan d'un appartement, plan d'une chambre, plan d'une table. La plupart des dossiers préparatoires contient de telles séries, plus ou moins complètes (La Curée, Le Ventre de Paris, Germinal, Une page d'amour, La joie de vivre, L'Assommoir, etc), où la précision peut aller jusqu'à la localisation des meubles dans une pièce (l'appartement d'Hélène dans Une page d'amour ${ }^{11}$ ). On peut lire, dans de telles séries, entre deux croquis, le travail de réajustement des scénarios (le passage d'une topographie centrée sur un château à une topographie centrée sur une église, dans le dossier préparatoire du Rêve $e^{12}$ ), la façon dont un plan pris sur nature au cours d'un «repérage » sur le terrain est redessiné pour aboutir à un nouveau plan "fictif» distribuant les habitats des personnages du roman (le plan de Cloyes devenant le plan de « Rognes », dans le dossier préparatoire de La Terre ${ }^{13}$ ), et, surtout, le souci de Zola de penser l'espace de son roman comme une hiérarchie d'espaces emboîtés, de sousespaces locaux intégrés à une hiérarchie globale, d'espaces régissant et d'espaces régis (les différents étages de la maison des Chanteau dans La joie de vivre, des Mouret pour La Conquête de Plassans, par exemple $\left.{ }^{14}\right)$. Cette pratique est à rapprocher, bien sûr, de certaines des théories zoliennes sur l'influence des milieux sur les personnes / personnages. Le personnage zolien est un être territorialisé, confiné à son espace, enserré et comme cadré par son habitat professionnel, volontiers assigné à résidence dans le lieu qu'il occupe, qui le conditionne, et qu'il est symétriquement chargé, selon le cahier des charges et les consignes les plus récurrentes des Ebauches et des Plans, de « rendre » de la façon la plus exhaustive et « documentaire » qui soit ${ }^{15}$. Les cloisons des appartements de la maison de La joie de vivre ou de Pot-bouille, de la «grande maison» de la Goutte d'Or, ou d'Une Page d'Amour, les «cases » du coron de Germinal ou du « carreau » des Halles, les étages et rayons du grand magasin (Le Louvre, Le Bon Marché), les murs d'enceinte de Plassans, de La Souléiade, ou du Paradou, le réseau des rues de tel ou tel quartier, sont là, selon deux figures graphiques dominantes de l'enfermement, le cercle d'une part, le carré d'autre part ${ }^{16}$, pour signifier un mode de destin régissant le personnel de l'œuvre à venir. Pour signifier, aussi, leur mode de vie, voué aux promiscuités du voisinage et de la contiguïté, aux flux des cancans et des commérages, thématique importante de l'œuvre zolienne. Pour signifier, enfin, une écriture, voire une rhétorique : la métonymie (figure de la contiguïté) et la synecdoque (figure de l'emboîtement), sont, selon plusieurs théoriciens (notamment Jakobson), les figures fondamentales de toute écriture réaliste.

8 La seconde figure topographique qui se laisse observer dans les dessins et croquis de Zola est celle du carrefour. Figure obsessionnelle de l'urbanisme de l'époque ${ }^{17}$, comme de la peinture de l'époque (Caillebotte, voir son grand tableau du Musée de Chicago), figure organisatrice de la ville haussmannienne du second Empire ${ }^{18}$, elle organise dans les dossiers préparatoires de Zola aussi bien les plans de lieux fictifs que ceux des lieux réels, de lieux urbains (les «quatre carrefours » de la place de la Bourse dans l'Argent) que de lieux ruraux, aussi bien les lieux « ouverts » (la plaine de Germinal, le champ de bataille de La Débâcle) que les lieux fermés (Plassans, le Paradou), notamment au niveau des croquis des lieux "globaux» qui organisent l'ensemble de la topographie d'un 
roman tout entier. Dans de tels plans Zola organise son espace de manière rayonnante et "cardinale", selon une sorte de "templum» originaire, autour d'une place (L'Argent), d'une " pointe» (St. Eustache, pour Le Ventre de Paris), d'un pont coupant un fleuve (L'Cuvre) ou un canal (Le Chesne, dans La Débâcle), d'un centre où convergent des ruisseaux (le Paradou), des routes, des sentiers et une rivière (Rognes, le village de La Joie de vivre), des rues (le carrefour rues Vineuse / Raynouard / de Passy d'Une Page d'Amour; le carrefour rue des Poissonniers / du faubourg Poissonnière / boulevard de Rochechouart / boulevard de La Chapelle de $L^{\prime}$ Assommoir ${ }^{19}$ ), une rue, ou une route, et une voie ferrée qui se "coupent " (La Bête humaine, au carrefour de L'Europe et à la Croix-de-Maufras), une route, une voie ferrée et un canal (Germinal), etc. Les rayons ainsi définis ont, aussi, une fonction de distribution des plantes (le Paradou), ou des classes (Plassans). Mais, contrairement aux plans par carrés et cercles, qui relèvent davantage (mais les deux systèmes coexistent, et ne sont pas incompatibles) d'un imaginaire de la localisation, de la clôture et de la distribution encyclopédique, ces croquis fondés sur un système cardinal centré mettent en place un système topographique plus dynamique, plus virtuellement narratif: les personnages se distribuent par rapport à un foyer central, par rapport à un point de convergence (d'où rencontres, conflits, "conquêtes" de lieux, circulations centripètes, antagonismes divers possibles, hésitations - le côté "Hercule à la croisée des chemins ») qui peut aussi devenir point de divergence (fuites, expulsions, circulations centrifuges, etc.). Des pôles antagonistes ou des nœuds de conflits s'y disposent et s'y opposent, en nébuleuse, définissant flux et reflux, par rapport à un point de rencontre central (l'église et la Halle, le coron et la maison des directeurs, l'église les Artaud et le Paradou, la maison et le cabaret, etc.). On est ici dans un imaginaire dynamique du réseau plus que dans un imaginaire cadastral du classement ou de la distribution ${ }^{20}$.
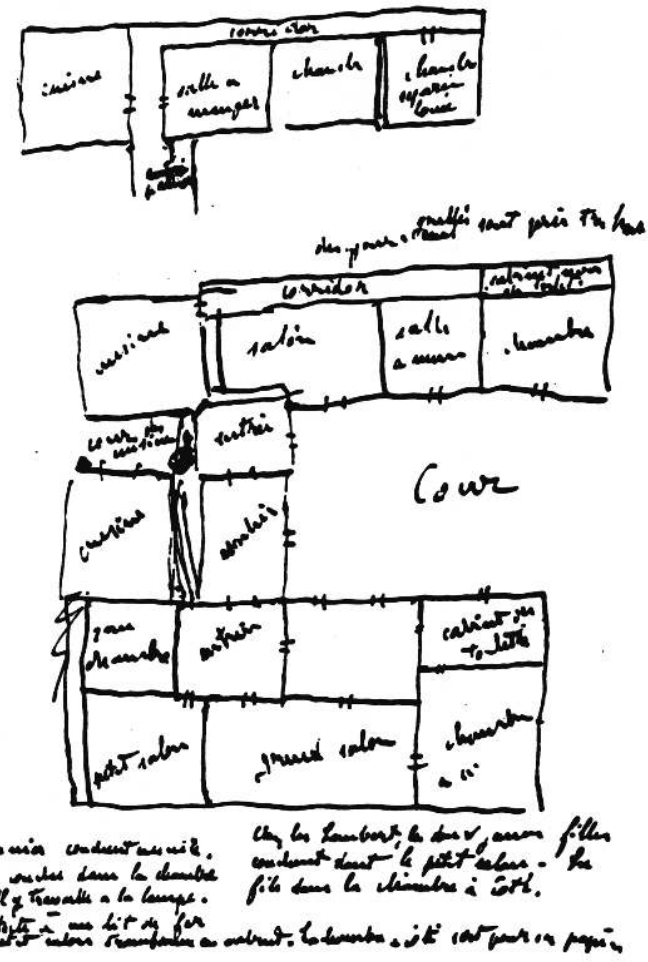

LE PREMIER ÉTAGE DE L'IMMEUBLE. PLAN AUTOGRAPHE D'ÉMILE ZOLA. DOSSIER MANUSCRIT DU ROMAN 


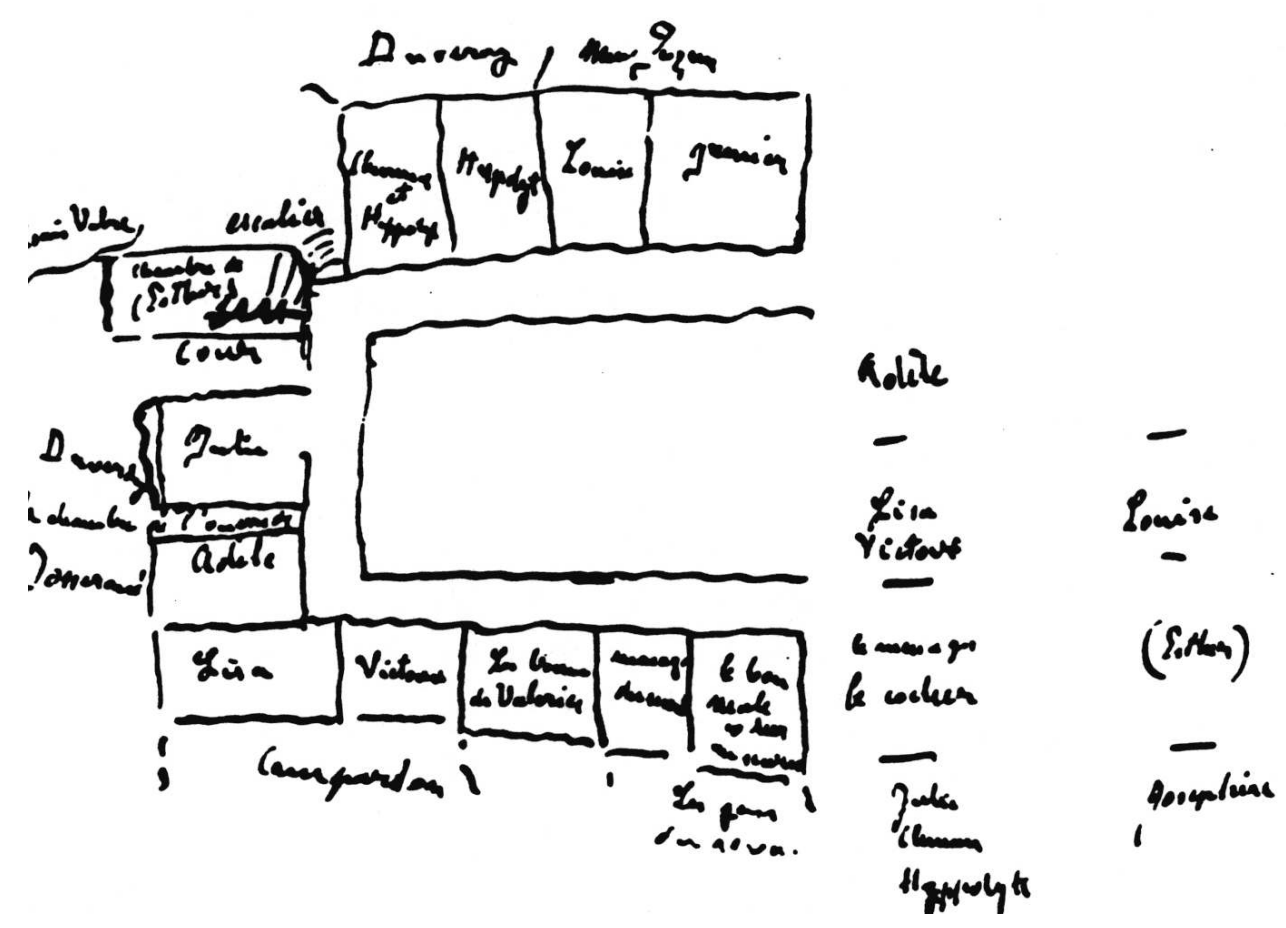

L'ÉTAGE DES CHAMBRES DE BONNES. PLAN D'ÉMILE ZOLA

L'analyse, que je n'ai pas le temps de développer ici, devrait bien sûr se fonder sur un examen plus exhaustif et plus minutieux de tous les croquis (de la main de Zola, ou de la main d'autres informateurs ${ }^{21}$ ) qui apparaissent dans les dossiers préparatoires. Il faudrait en construire une typologie plus fine qui tienne compte des petits croquis insérés au fil même du texte préparatoire (voir les deux exemples évoqués en note 9), interpréter les ratures d'espace qui s'y manifestent, les remords et repentirs graphiques de Zola (pourquoi ce «détour " ajouté au chemin de Plassans à la Palud pour le faire contourner le village des Artaud, et non plus le traverser, contrairement à une première solution que Zola a biffée ?22), analyser les croquis qui paraissent atypiques (le plan "éparpillé » de La Souléiade, dont la logique schématique n'apparait pas nettement à première lecture - mais une voie de chemin de fer la jouxte, et un mur d'enceinte l'entoure : espace "tangentiel $»^{23}$ ), analyser leur mode de relation (lequel paraphrase l'autre ?) avec les notes « de repérage » rédigées par Zola sur le terrain, et essayer de localiser leur moment précis d'apparition dans la durée de l'élaboration de l'œuvre. L'arbre (-généalogique - qui sollicite l'imaginaire des filiations, des causalités), le cercle et le carré (qui sollicite l'imaginaire taxinomique des emboîtements, des enfermements, des voisinages et des contiguïtés), et le "templum » (qui sollicite l'imaginaire scénarique de la mise en intrigue des conflits), sont sans doute les figures spatiales majeures, à la fois incitatrices et régulatrices, et non incompatibles ${ }^{24}$, de l'écriture zolienne au cours de sa production et de son engendrement. C'est, dans l'avant-texte des œuvres, le jeu réciproque de tels schèmes (plus que le jeu plus apparent et plus « lisible» des thèmes explicités) qui devrait faire l'objet d'une véritable analyse génétique. 


\section{NOTES}

1. La formule est du Docteur Édouard Toulouse, dans son Enquête médico-psychologique sur les rapports de la supériorité intellectuelle avec la névropathie, Introduction Générale, Emile Zola, Paris, Société d'Editions scientifiques, 1896, p. 269. Le Docteur Toulouse traite des «Procédés de composition » de son « patient " page 268 et suivantes.

2. Le Docteur Toulouse note en particulier l'habitude qu'a Zola de tracer « quand il le faut, des plans d'appartements et d'autres lieux » (ouvr. cit. p. 272)

3. Aucune de ces entités sémiotiques, bien sûr, n'est incompatible avec les autres, et les linguistes savent que les signes (discrets, arbitraires) du langage peuvent recevoir une fonction icônique (analogique, motivée) : voir R. Jakobson: «A la recherche de l'essence du langage ", Diogène, Problèmes du langage, Gallimard, $\mathrm{n}^{\circ}$ 51, 1966.

4. Voir l'inventaire des tableaux, gravures et sculptures possédés par Zola et dispersés à sa mort (Catalogue reproduit par C.Becker dans : Dictionnaire d'Emile Zola, Paris, Laffont, «Bouquins », p. 643 et suiv.).

5. Voir John Grand-Carteret : Zola en images, Paris, Félix Juven, 1905, et les actes d'un colloque, «Zola en images", réunis dans un numéro spécial des Cahiers Naturalistes, n 66, 1992. Curieusement, aucune des études réunies dans ce numéro spécial, pourtant attentif à jeter les bases d'une « iconothèque » zolienne générale, ne traite de la question (l'image, les dessins de la main de Zola, en avant-texte) que j'aborde ici. Même chose pour un collectif : L'image génératrice de textes de fiction (sous la direction de P.Mourier-Casile et D. Maucond'huy), Poitiers, La Licorne, s.d. Une publication exhaustive des dessins de Zola reste à entreprendre.

6. Voir, pour ne citer que deux exemples de textes accompagnés de dessins de l'auteur, Le Rhin de V. Hugo, ou le Brulard de Stendhal.

7. Sur la composante intertextuelle à l'œuvre dans une Ebauche de Zola (celle de La Bête humaine), voir mon article : «Echos et reflets » dans Poétique n 109, 1997.

8. Un certain nombre d'entre eux a été publié et reproduit par H. Mitterand et J. Vidal dans l'Album Zola (Paris, Gallimard, Bibliothèque de La Pléiade, 1963), ou dans l'anthologie des dossiers préparatoires de Zola établie par H. Mitterand: Emile Zola, Carnets d'enquête, une ethnographie inédite de la France, Paris, Plon, 1986. Ces dessins sont répertoriés, et quelques uns reproduits, dans les notes de H. Mitterand à son édition des Rougon-Macquart (Paris, Gallimard, Bibliothèque de La Pléiade, 5 volumes, 1960-1967). Voir pages suivantes.

9. Quelques exceptions : un petit dessin, dans le fil du texte des notes, pour les poutres de l'église de Romilly sur Aigre (dossier préparatoire de La Terre, reproduit par H. Mitterand p. 576 dans ses Carnets d'Enquêtes, ouvr. cit), ou un petit schéma (trois piquets) dans les notes prises pour un jeu d'estaminet, le «billon », dans le dossier de Germinal. Voir aussi les coupes d'un puits de mine (dossier de Germinal ; voir les dessins de ce dossier reproduits par C. Becker dans Emile Zola: la fabrique de Germinal, Paris S.E.D.E.S., 1986, p. 478, et p. 335 et suiv.), et la façade du théâtre des Variétés (dossier préparatoire de Nana)

10. Une exception notable, et intéressante pour étudier le travail de l'imaginaire (et de la « mise en abîme ») chez Zola, son dessin du blason des Hautecœur (qu'il invente) dans le dossier préparatoire du-Rêve.

11. Reproduit par H. Mitterand dans son édition des Rougon-Macquart de la Bibiothèque de La Pléiade, ed. cit. tome II, p. 1618. La chaise longue d'Hélène y est notée, meuble emblématique de la rêveuse héroine. Voir aussi le croquis de la chambre où a couché Napoléon III sur le champ de bataille (Dossier préparatoire de La Débâcle, reproduit dans Carnets d'enquetes, ouvr. cit., p. 646).

12. Les deux plans sont reproduits par H. Mitterand au tome IV de son édition des RougonMacquart de la Bibliothèque de La Pléiade (ed. cit. p. 1640 et p. 1641). Le dessin du blason des 
Hautecœur, qu'invente et dessine Zola, paraphrase en quelque sorte cette hésitation du scénario, en juxtaposant, « écartelé », selon donc une sorte de "templum » (voir ci-après) héraldique, un château et une croix.

13. Ibid., p. 1515 et 1516.

14. Album Zola (ouvr.cit) p. 133.

15. Sur le cahier des charges, essentiellement descriptif, documentaire et sociologique, du projet zolien, voir Ph. Hamon: Le personnel du roman (Genève, Droz, 1983) et Du descriptif (Paris, Hachette, 1993). Le terme de «cadre " revient en leitmotiv sous la plume de Zola décrivant, en 1868, pour l'éditeur Lacroix et pour lui-même, les futurs romans de la série qu'il projette.

16. Voir notamment les plans circulaires (ou ovoïdes) de Plassans, de La Souleiade, et du Paradou, par opposition aux plans «cadastraux ", par carrés et rectangles, des appartements parisiens. Zola, préfaçant le premier roman de sa série, La Fortune des Rougon, écrit que son œuvre «s'agite dans un cercle fini » (Les Rougon-Macquart, ed. cit. tome I, p. 4). Commencée dans une ville close, Plassans, la série se terminera dans une ville encerclée (Sedan, dans La Débâcle). Le plan de la serre de La Curée représente un bassin et des massifs circulaires inscrits dans une forme générale carrée (reproduit dans Carnets d'enquêtes, ouvr. cit. p.33). Même dispositif, en gros, pour le jardin du docteur Deberle, dans le dossier d'Une Page d'Amour (reproduit dans l'édition des RougonMacquart de la Bibliothèque de La Pléiade, tome II p. 1617).

17. Voir notamment Eugène Hénard (1849-1923), architecte de la ville de Paris: Etudes sur les transformations de Paris et autres écrits sur l'urbanisme (1903-1909), présentation par J. L. Cohen, Paris, L'Equerre, 1982.

18. Voir Fr. Paul-Lévy : La ville en croix, Paris, Librairie des Méridiens, 1984. Toute l'intrigue de La Curée est axée sur la spéculation immobilière qui se déchaine à l'occasion du percement de «la grande croisée de Paris ». La formule, de Haussmann, se retrouve dans la bouche de Saccard (Les Rougon-Macquart, ed. cit., tome I, p. 389 : « De sa main étendue, ouverte et tranchante comme un coutelas, il fit signe de séparer la ville en quatre parts (...) La grande croisée de Paris, comme ils disent »).

19. Voir les deux croquis publiés par H. Mitterand dans: Carnets d'enquêtes (ouvr. cit. p. 418). L'Assommoir du père Colombe est évidemment situé au carrefour. Et remarquons, jouxtant ce carrefour principal, la croix de St André formée par les rues de Chartres et Charbonnières, qui a peut-être attiré l'œil de Zola sur ce quartier de Paris.

20. Le «templum » comme schème organisateur des romans zoliens a fait l'objet d'une bonne première approche par 0 . Lumbroso : «La figure du croisement dans l'œuvre d'Emile Zola ", Cahiers Naturalistes $n^{\circ} 67,1993$. Mes réflexions se situent donc en complémentaire (l'avant-texte) de son étude, qui porte surtout sur une mise en place théorique de la notion, et sur l'œuvre (publiée) de Zola. Le «templum» a fait l'objet d'une importante théori-sation, plus logique et globalement cognitive, par Pierre Boudon : Le paradigme de l'architecture (Balzac, Candiac, Canada, 1992). Plus généralement, les littéraires gagneraient certainement à regarder du côté des théoriciens de l'architecture qui se sont intéressés aux modes de conception et de représentation de l'espace bâti. Voir, outre les travaux de Philippe Boudon (à ne pas confondre avec Pierre!), bien connus, comme : Sur l'espace architectural (Paris, Dunod, 1971) : C. Camus : Lecture sociologique de l'architecture décrite, comment bâtir avec des mots?, Paris, L'Harmattan, 1996; F. Pousin: L'architecture mise en scène, Paris, Arguments, 1995. Voir aussi le collectif: Testo letterario e immaginario architettonico, a cura di R. Casari, M. Lorandi, U. Persi, F. R. Amaya, Jaca Book, Milan, 1996.

21. Un plan de la Bourse dans le dossier de L'Argent, des plans des pavillons des Halles dans celui $\mathrm{du}$ Ventre de Paris, des détails des puits dans le dossier de Germinal, ne sont pas de la main de Zola.

22. Dessin reproduit dans l'Album Zola (ouvr. cit), p. 135. Zola écrit pour lui-même en marge : « Le chemin de Plassans à La Palud passe à une lieue des Artauds». On passe d'un imaginaire de la 
" coupure " (un chemin "coupe» un village, le traverse, le met en communication) à un imaginaire du contournement, de l'encerclement, de l'isolement.

23. Reproduit dans Carnets d'enquêtes, ouvr. cit, p. XII du folio de planches central.

24. Un croquis pour La Terre combine par exemple les accolades «en arbre » de la division des parcelles et du morcellement des propriétés avec des croquis «cadastraux » (reproduit dans l'Album Zola (ouvr. cit.) p. 227.

\section{AUTEUR}

\section{PHILIPPE HAMON}

Université Paris III - Sorbonne Nouvelle 